This work is on a Creative Commons Attribution 4.0 International (CC BY 4.0) license, https://creativecommons.org/licenses/by/4.0/. Access to this work was provided by the University of Maryland, Baltimore County (UMBC) ScholarWorks@UMBC digital repository on the Maryland Shared Open Access (MD-SOAR) platform.

Please provide feedback

Please support the ScholarWorks@UMBC repository by emailing scholarworks-group@umbc.edu and telling us what having access to this work means to you and why it's important to you. Thank you. 


\title{
An introduction to using counterdiabatic driving to eliminate genetic lag in changing environments
}

\author{
Emily Dolson ${ }^{1, *}$, Shamreen Iram ${ }^{2, *}$, Joshua Chiel ${ }^{2, *}$, Julia Pelesko', \\ Nikhil Krishnan ${ }^{3}$, Özenç Güngör ${ }^{2}$, Benjamin Kuznets-Speck ${ }^{2,4}$, Sebastian Deffner ${ }^{5}$, Efe Ilker ${ }^{6}$, \\ Jacob G. Scott ${ }^{1,2,3}$ and Michael Hinczewski ${ }^{2}$ \\ ${ }^{1}$ Translational Hematology Oncology Research, Cleveland Clinic, Cleveland OH, 44106, USA \\ ${ }^{2}$ Department of Physics, Case Western Reserve University, Cleveland, OH, 44106, USA \\ ${ }^{3}$ Case Western Reserve University School of Medicine, Cleveland, OH, 44106, USA \\ ${ }^{4}$ Biophysics Graduate Group, University of California, Berkeley, CA 94720, USA \\ ${ }^{5}$ Department of Physics, University of Maryland, Baltimore County, Baltimore, MD 21250, USA \\ ${ }^{6}$ Physico-Chimie Curie UMR 168, Institut Curie, PSL Research University, 75248 Paris Cedex 05, France \\ * denotes equal contribution emilyldolson@gmail.com
}

\section{Introduction}

Evolution is increasingly recognized as a powerful tool for building and controlling living and lifelike systems. However, the complexity and stochasticity of the evolutionary process have thus far made it more of a blunt instrument than a tool that can be precisely controlled. Here, we present an approach that can begin to give us the level of fine control we need to harness evolution for a wider variety of purposes. A common step in using evolution to achieve a desired end result is to gradually change an environment over time along some continuous axis. Currently, our lack of rigorous theory predicting the rate at which this adaptation will occur means a population must be repeatedly tested to know when it has reached the desired end state. This requirement is not ideal, as there are some contexts in which such testing is impossible (e.g. measuring the current genetic state of drug-resistant cancer cells in a patient's body). Here, we describe the beginning of a solution to this problem (presented in full in (Iram et al., 2019)). As a starting point, we consider an ideal case where we have perfect knowledge of the fitness landscape, frequent mutations, and a large population. Using an approach from physics called counterdiabatic (CD) driving, we can mathematically derive a prescription for how to change the environment so that the population arrives at the adaptive end-state at a fast and predictable speed.

$\mathrm{CD}$ driving is a concept originally developed in quantum mechanics Demirplak and Rice (2003, 2005); Berry (2009). More recently, it has been used in classical physics applications such as the creation of an optical tweezer Martínez et al. (2016). Fundamentally, CD driving is a way to force a noisy system to equilibrate to a new state arbitrarily quickly by subjecting it to a different series of intermediate states. As an intuitive example, consider a waiter carrying a glass of water on a tray (Sels and Polkovnikov, 2017). If he keeps the tray perfectly flat while walking, he will need to move very slowly to avoid spilling any water. He can walk much more quickly without spilling if he instead tilts the tray slightly to counteract the force his acceleration exerts on the glass. In so doing, he has used a CD force - a new degree of freedom that can be manipulated to move a system more quickly between a given start and end state.

\section{Counterdiabatic Driving of Evolution}

Like the glass of water, a population undergoing natural selection will have a predictable equilibrium in a given environment, but will be behave unpredictably if the environment is rapidly perturbed. As a concrete example, we consider a population in an empirically-measured fitness landscape based on antimalarial drug resistance genes Ogbunugafor et al. (2016); Brown et al. (2010). If the population remains in a given environment (defined here as a specific drug concentration) long enough, it will reach an equilibrium. Most of the population will have a genotype corresponding to the highest point(s) in the fitness landscape for that environment. Depending on the balance of mutation and selection, mutationally-adjacent genotypes will also be present at some frequency.

Adding some quantity of a drug will put the population into a new fitness landscape. This new landscape also has an equilibrium genotype distribution, which the population will reach eventually. However, this equilibration process could potentially take a long time. A scenario where the drug dosage increases over time (common in medicine) results in applying a sequence of fitness landscapes much more rapidly than the population can reach the equilibrium genotype distribution for any of them. As a result, we see a "lag" between the current genotype distribution and the equilibrium distribution for the current drug concentration. 


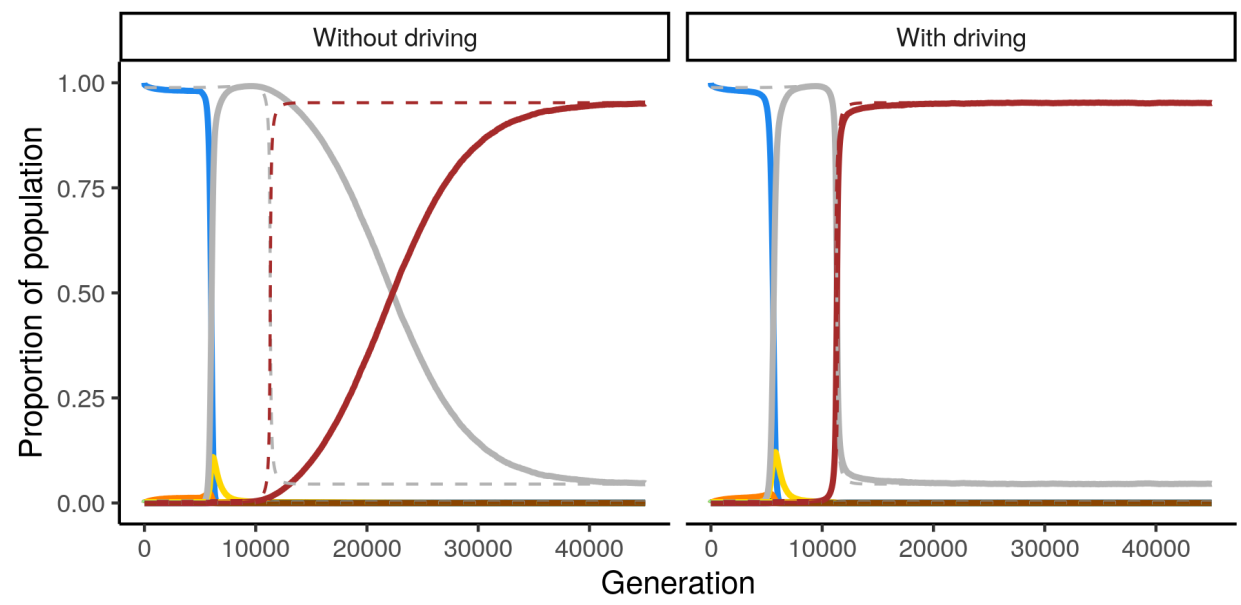

Figure 1: CD driving eliminates evolutionary lag in 16-genotype simulation. Change in genotype frequencies over a period of environmental change (increasing drug concentration). Each color shows a different one of the 16 genotypes included in an agent based model. Dashed lines indicate the theoretical equilibrium value at each point in time, based on the current drug concentration. Solid lines indicate the observed value (median out of 10 replicates; error bars are plotted but are too small to see). Without CD driving, the observed values (solid lines) lag substantially behind the equilibrium values (dashed lines). CD driving almost completely eliminates this lag.

We can eliminate this lag using a $\mathrm{CD}$ driving protocol based on our drug ramp-up pattern (see Figure 1; for the math used to create the protocol, see (Iram et al., 2019)). This protocol will be a sequence of drug doses to administer. By following the protocol, we can force the genotype distribution in the population to always match the equilibrium distribution for the original drug ramp-up pattern (see Figure 1). As a result, we are able to move the population through the fitness landscape at a predictable speed.

\section{Conclusion}

We have shown that using CD driving to control the speed with which a population traverses a fitness landscape works. Our protocol is relatively robust to errors in parameter estimates and constraints on the maximum drug concentration (Iram et al., 2019). Note that drug concentrations are just one example of a selection pressure that can be varied over time. Currently the applications for this work are limited by the lack of systems for which we have sufficiently quantified the relevant portion of the fitness landscape. However, we anticipate more fitness landscapes being measured in the near future. When combined with ongoing work on using environmental sequencing to steer evolution (Nichol et al., 2015; Maltas and Wood, 2018), we believe CD driving will help us gain more control over evolving systems.

\section{References}

Berry, M. V. (2009). Transitionless quantum driving. J. Phys. A: Math. Theor, 42(36):365303.

Brown, K. M., Costanzo, M. S., Xu, W., Roy, S., Lozovsky, E. R., and Hartl, D. L. (2010). Compensatory mutations restore fit- ness during the evolution of dihydrofolate reductase. Mol. Biol. Evol., 27(12):2682-2690.

Demirplak, M. and Rice, S. A. (2003). Adiabatic population transfer with control fields. J. Phys. Chem. A, 107(46):9937-9945.

Demirplak, M. and Rice, S. A. (2005). Assisted adiabatic passage revisited. J. Phys. Chem. B, 109(14):6838-6844.

Iram, S., Dolson, E., Chiel, J., Pelesko, J., Krishnan, N., Özenç Güngör, Kuznets-Speck, B., Deffner, S., Ilker, E., Scott, J. G., and Hinczewski, M. (2019). Controlling the speed and trajectory of evolution with counterdiabatic driving.

Maltas, J. and Wood, K. B. (2018). Pervasive and diverse collateral sensitivity profiles inform optimal strategies to limit antibiotic resistance. bioRxiv, page 241075.

Martínez, I. A., Petrosyan, A., Guéry-Odelin, D., Trizac, E., and Ciliberto, S. (2016). Engineered swift equilibration of a brownian particle. Nat. Phys., 12(9):843.

Nichol, D., Jeavons, P., Fletcher, A. G., Bonomo, R. A., Maini, P. K., Paul, J. L., Gatenby, R. A., Anderson, A. R., and Scott, J. G. (2015). Steering evolution with sequential therapy to prevent the emergence of bacterial antibiotic resistance. PLoS Comp. Biol., 11(9):e1004493.

Ogbunugafor, C. B., Wylie, C. S., Diakite, I., Weinreich, D. M., and Hartl, D. L. (2016). Adaptive landscape by environment interactions dictate evolutionary dynamics in models of drug resistance. PLoS Comp. Biol., 12(1):e1004710.

Sels, D. and Polkovnikov, A. (2017). Minimizing irreversible losses in quantum systems by local counterdiabatic driving. Proceedings of the National Academy of Sciences, 114(20):E3909-E3916. 